\title{
Diversité génétique de quelques populations naturelles de Medicago ciliaris (L) Krock et de Medicago intertexta (L) Mill. I. Analyse de la variabilité morphologique
}

\author{
K Cherifi ${ }^{*}$, M Boussaïd 2, M Marrakchi 1 \\ 1 Laboratoire de génétique moléculaire, faculté des sciences, Tunis; \\ 2 Laboratoire de botanique, faculté de pharmacie, Monastir, Tunisie
}

(Reçu le 9 avril 1993; accepté le 11 août 1993)

\begin{abstract}
Résumé - L'évaluation de la diversité génétique a porté sur 11 populations naturelles de Medicago annuelles, originaires du Nord de la Tunisie : 8 populations de $M$ ciliaris et 3 populations de $M$ intertexta. Vingt caractères morphologiques se rapportant au développement végétatif et reproducteur des plantes cultivées dans des conditions contrôlées ont été retenus dans cette étude. L'analyse canonique discriminante et la distance de Mahalanobis ont révélé au sein de chaque taxon une diversité intra- et interpopulations importante. Une distinction relative nette entre les groupes des populations des 2 espèces a été également mise en évidence. Toutefois une population de $M$ intertexta semble présenter des similarités morphologiques avec celles de $M$ ciliaris. Cette situation particulière traduirait la possibilité d'hybridations introgressives entre ces 2 taxons.
\end{abstract}

variabilité morphologique / introgression / Medicago ciliaris / Medicago intertexta / canonique discriminante

Summary - Genetic diversity in some natural populations of $M$ ciliaris (L) Krock and M intertexta (L) Mill. I. Analysis of morphological variability. Genetic diversity was studied in 11 wild populations of the annual Medicago in northern Tunisia: 8 populations of $\mathrm{M}$ ciliaris and 3 populations of $\mathrm{M}$ intertexta. Twenty morphological characters, related to vegetative and reproductive development of plants cultivated in controlled conditions, were studied. Canonical discriminant analysis and Mahalanobis's distance showed an important variability within and between populations, and a net separation between population groups of the 2 species. However, one population of $\mathrm{M}$ intertexta seems to present morphological similarity with $\mathrm{M}$ ciliaris. This particular situation suggests the possibility of introgressive hybridization between these 2 taxa.

morphological variability / introgression / Medicago ciliaris / Medicago intertexta / canonical discriminant

\section{INTRODUCTION}

Les ressources génétiques des populations naturelles de diverses espèces végétales constituent un patrimoine d'une grande importance. L'étude et la préservation de la diversité génétique des espèces sauvages sont indispensables à l'amélioration des espèces domestiquées pour faire face à de nouveaux impératifs. Dans le cas des espèces fourragères et pastorales, plus particulièrement les Medicago annuelles, plusieurs travaux de sélection variétale utilisant la diversité génétique des populations naturelles ont été menés par les chercheurs australiens depuis 1950. II existe à présent plus d'une vingtaine de cultivars de ces espèces, répondant à diverses conditions pédo-climatiques (Gintzburger et Prosperi, 1987).

L'analyse de la diversité génétique des espèces sauvages a permis également d'élucider différents problèmes de taxonomie et de relations phylogénétiques, ainsi que l'organisation

\footnotetext{
* Correspondance et tirés à part
} 
de la variabilité génétique des populations de diverses espèces de Légumineuses fourragères ou à graines des genres Médicago (Damerval, 1983 ; Elmossadik, 1991 ; Small et Fawzy, 1992), Hedysarum (Baâtout et al, 1991), Phaseolus (Singh et al, 1991).

Le présent travail porte sur 2 espèces annuelles diploïdes $(2 n=16)$ du genre Medicago: $M$ ciliaris ( $L$ ) et $M$ intertexta ( $L$ ). Ces espèces, préférentiellement autogames (Lesins et Lesins, 1979), poussent spontanément en Tunisie, dans des étages bioclimatiques allant de l'humide à l'aride supérieur. Elles constituent d'excellentes plantes de pâture, et semblent tolérer la salinité (Rouz, 1991). La difficulté d'identification de ces 2 espèces, étant donné leurs ressemblances morphologiques, a été l'origine de nombreuses confusions taxonomiques (Small et Jomphe, 1989). L'angle d'insertion des épines sur la gousse est souvent considéré comme le caractère botanique principal différenciant les 2 espèces (Pottier-Alapetite, 1979; Lesins et Lesins, 1979; Small et Jomphe, 1989).

L'objectif essentiel de cette étude consiste en une analyse de la diversité morphologique de populations naturelles, cultivées en conditions contrôlées, à travers 20 caractères biométriques.

\section{MATÉRIEL ET MÉTHODES}

En Tunisie, $M$ ciliaris est localisée au niveau des étages bioclimatiques allant de l'humide inférieur à l'aride supérieur (Abdelkefi et al, 1992). L'espèce est abondante dans les étages sub-humide et semi-aride inférieur, sous une pluviométrie moyenne annuelle comprise entre 400 et $600 \mathrm{~mm}$. Toutefois, PottierAlapetite (1979) l'a signalée comme adventice accompagnant les cultures céréalières, dans l'étage aride inférieur (régions de Gabes, El-Guetar). Medicago intertexta semble être moins fréquente et occupe des aires plus restreintes. Elle ne dépasse pas l'étage bioclimatique semi-aride supérieur. Elle se localise dans les zones à pluviométrie annuelle supérieure à $550 \mathrm{~mm}$ et à des altitudes comprises entre 100 et $200 \mathrm{~m}$.

Les 11 populations naturelles englobant les 2 espèces ont été prospectées vers les mois de juin et juillet 1991. Elle ont été choisies en fonction des étages bioclimatiques où les 2 espèces sont les mieux représentées. Il s'agit des étages sub-humide et humide pour $M$ intertexta, sub-humide et semi-aride pour $M$ ciliaris. Dans certains cas, nous avons multiplié les sites d'échantillonnage au sein d'un même sous-étage bioclimatique pour tenter d'identifier des génotypes particuliers.

Pour chaque taxon, des prélèvements d'échantillons de gousses (une centaine), représentatifs de chaque population, ont été ramassés au hasard. La collecte a été effectuée dans un espace le plus vaste possible pour avoir un échantillon rassemblant le maximum de diversité génétique.

Les 8 populations de $M$ ciliaris sont originaires des régions de Beja (Bej), Bizerte (Biz), Tunis (Tun), Jenbouda (Jen), Medjez-Elbab (Mjb), Sebkhat-Bouficha (Seb), Menzel-Bourguiba (Meb) et Korba (Kor). Celles de $M$ intertexta sont collectées près de Sedjnane (Sed), Mateur (Mat) et Aïn-Dragam (Aïd). La localisation géographique et les étages bioclimatiques auquels appartiennent ces populations figurent sur la carte (fig 1).

\section{Conditions de culture}

Des graines issues de différentes gousses, prélevées au hasard au sein des échantillons collectés dans la nature, sont scarifiées puis mises à germer dans les boîtes de Pétri sur papier filtre imbibé d'eau. Cinq j après la mise en germination, les plantules sont transférées isolément en pots $(20 / 25 \mathrm{~cm})$, contenant tous une même terre argilo-calcaire préalablement tamisée et non traitée. Les plantes sont cultivées sous abri (serre tunnel en plastique de $6 \mathrm{~m}$ de large et $12 \mathrm{~m}$ de long) dans les conditions environnementales de la région de Tunis. Un arrosage à l'eau de ville est effectué quotidiennement.

Les 11 populations considérées ont été traitées sous forme de 3 répétitions en blocs $(3,5 / 3,5 \mathrm{~m})$. Une bande de $1,5 \mathrm{~m}$ sépare 2 blocs successifs. Chaque répétition contient 11 populations randomisées, représentée chacune par 10 individus tirés au hasard et disposés en ligne.

Nous avons suivi la morphogenèse des plantes depuis la germination (mois de septembre) jusqu'à la maturation des fruits (mois de mai-juin).

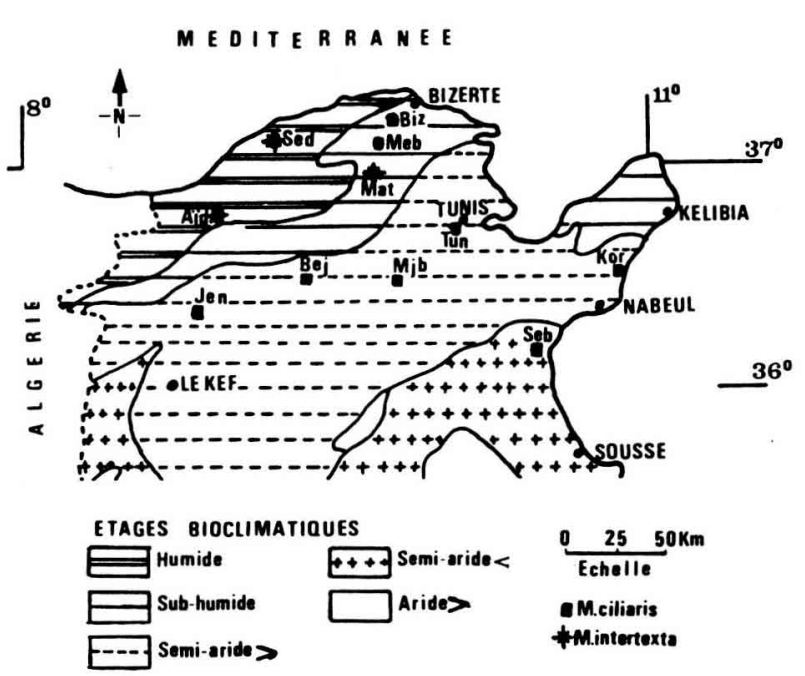

Fig 1. Carte de la Tunisie du Nord : étages bioclimatiques et localisation des populations de $M$ ciliaris et de $M$ intertexa analysées. 
Vingt caractères mesurés sur l'appareil aérien (végétatif et reproducteur) ont été retenus pour analyser la variabilité génétique (tableau I). La plupart de ces caractères figurent dans le manuel de descripteurs pour Medicago annuelles de I'IBPGR (International Board for Plant Genetic Ressources) (IBPGR, 1991).

\section{Méthodes statistiques utilisées}

Pour comparer les populations d'une même espèce ou des 2 espèces prises simultanément sur la base des 20 caractères considérés, nous avons effectué une analyse de variance à 2 critères de classification (modèle aléatoire avec effet bloc et effet population). La structuration de la variabilité des populations d'une même espèce selon les conditions écogéographiques des stations d'origine et la divergence morphologique interspécifique ont été abordées par l'analyse canonique discriminante (can-disc). Cette dernière est basée sur les travaux de Fisher (1936) et a été depuis largement utilisée pour l'analyse de caractères quantitatifs, mesurés sur des populations d'une même espèce ou d'espèces différentes (Rao,

Tableau I. Caractères mesurés.

\section{Code}

Signification

DF1 Date d'apparition de la 1 re feuille (j)

DF2 Date d'apparition de la $2^{e}$ feuille (j)

DF3 Date d'apparition de la $3^{e}$ feuille (j)

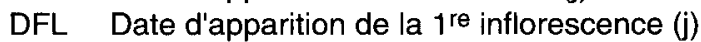

PRC Nombre de feuilles avant la $1^{\text {re }}$ inflorescence sur le plus long rameau plagiotrope

LO Longueur de l'axe orthotrope à la fin du cycle de développement $(\mathrm{cm})$

NIO Nombre total d'inflorescences portées par l'axe orthotrope

LRL Longueur (cm) du plus long rameau plagiotrope en fin de végétation

LRX Longueur $(\mathrm{cm})$ de tous les rameaux latéraux plagiotropes

NIRL Nombre d'inflorescences sur le plus long rameau

IXF Nombre maximum de fleurs par inflorescence

NRX Nombre des axes plagiotropes formés en fin de végétation

MFI Nombre moyen de fleurs par inflorescence

LIF6 Long/larg de la foliole principale de la $6^{e}$ feuille

TF6 Taille de la foliole principale de la $6^{e}$ feuille ( $A$ Log $A$ où $A=$ Long*larg)

NSG Nombre de spires de la gousse

NFO Nombre de feuilles sur l'axe orthotrope

HG Hauteur de la gousse (cm)

$D G$ Diamètre de la gousse $(\mathrm{cm})$

HDG Hauteur/diamètre de la gousse
1972 ; Hebrant, 1975 ; Semple et al, 1991 ; Robert et Sarr, 1991).

Elle cherche des variables canoniques non corrélées: combinaisons linéaires des paramètres observés. La procédure can-disc (programme SAS) utilisée fournit en plus d'une représentation graphique des différentes populations dans le plan défini par les diverses composantes canoniques prises 2 à 2, les distances de Mahalanobis calculées par la formule suivante :

$$
D^{2}(i j)=\left(x_{i}-x_{j}\right)^{\prime} W^{-1}\left(x_{i}-x_{j}\right)
$$

où $W$ est la matrice de variance-covariance intrapopulation, $x_{i}$ et $x_{i}$ sont les vecteurs d'observation des populations $i$ et $j$. Un test $F$ de Fisher et Snedecor peut être calculé, sous l'hypothèse qu'il n'y a pas de différence significative entre les populations $i$ et $j$.

$$
F=\frac{n_{i} \cdot n_{j}\left(\left(n_{i}+n_{j}-2\right)-(p-1)\right)}{p\left(n_{i}+n_{j}\right)\left(n_{i}+n_{j}-2\right)} \cdot D^{2}{ }_{i j}
$$

$p$ étant le nombre de caractères observés; $n_{i}$ et $n_{i}$ sont les effectifs des populations $i$ et $j$.

L'ensemble de ces calculs a été réalisé sur ordinateur par le programme SAS (Statistical analysis system) (SAS, 1990), avec les procédures ANOVA et CANDISC.

\section{RÉSULTATS}

\section{Analyse des caractères pris séparément}

L'analyse de variance à 2 critères de classification (population et bloc) effectuée sur les populations d'une même espèce ou pour les 2 espèces considérées ensemble révèle un effet population hautement significatif pour la majorité des variables utilisées (tableaux II, III, IV). Quant à l'effet bloc, il n'est significatif que pour le caractère DFL (date de floraison) dans le cas de $\mathrm{M} \mathrm{ci-}$ liaris et l'ensemble des populations des 2 espèces. II semblerait donc que la mise à fleur soit sensible à de très faibles variations du milieu (température), qui peuvent se manifester dans une serre tunnel en plastique.

Le nombre et la nature des caractères présentant des variations significatives diffèrent selon l'espèce considérée. Chez Medicago ciliaris, seul le caractère L1F6 (rapport longueur sur largeur de la foliole principale de la sixième feuille) semble être homogène entre les populations (tableau I). L'hétérogénéité des caractères entre les populations de Medicago intertexta est légèrement moins importante puisque 5 caractères 
Tableau II. Résultats de l'analyse de variance à 2 critères de classification (modèle aléatoire) des différents caractères étudiés chez les populations de $M$ ciliaris.

\begin{tabular}{|c|c|c|c|c|c|}
\hline Caractères & $C M_{p o p}$ & $C M_{b l o c}$ & $C M_{i n t}$ & $\begin{array}{c}F_{p o p} 7 / 14 \\
\left(C M_{p o p} / C m_{i n t}\right)\end{array}$ & $\begin{array}{l}F_{b l o c^{2 / 14}} \\
\left(C M_{p o p} / C m_{i n t}\right)\end{array}$ \\
\hline DF1 & 64,55 & 3,61 & 1,65 & $39,12^{\star *}$ & $2,17 \mathrm{NS}$ \\
\hline DF2 & 223,01 & 8,32 & 9,21 & $24,21^{\star *}$ & $0,90 \mathrm{NS}$ \\
\hline DF3 & 456,71 & 5,27 & 7,74 & $59,00^{\star *}$ & $0,68 \mathrm{NS}$ \\
\hline DFL & 3297,49 & 1204,73 & 278,73 & $11,83^{\star \star *}$ & $4,32^{*}$ \\
\hline PRC & 202,17 & 2,004 & 7,93 & $25,49^{\star \star}$ & $0,25 \mathrm{NS}$ \\
\hline LO & 1204,68 & 113,65 & 136,79 & $8,80^{* *}$ & 0,83 NS \\
\hline $\mathrm{NIO}$ & 14,04 & 1,21 & 2,28 & $6,15^{\star \star}$ & 0,53 NS \\
\hline LRL & 4305,68 & 484,73 & 219,55 & $19,61^{\star *}$ & $2,20 \mathrm{NS}$ \\
\hline LRX & 203830,23 & 7948,61 & 12663,34 & $16,09^{\star \star}$ & $0,62 \mathrm{NS}$ \\
\hline NIRL & 23,72 & 7,23 & 2,50 & $9,49^{\star \star}$ & $2,88 \mathrm{NS}$ \\
\hline IXF & 1,80 & 0,45 & 0,15 & $12,00^{\star *}$ & $3,00 \mathrm{NS}$ \\
\hline NRX & 36,00 & 4,38 & 4,13 & $8,71^{\text {** }}$ & $1,06 \mathrm{NS}$ \\
\hline $\mathrm{MFI}$ & 3,41 & 0,12 & 0,13 & $26,23^{\star \star}$ & $0,87 \mathrm{NS}$ \\
\hline NSG & 8,54 & 0,03 & 0,16 & $53,37^{\star \star}$ & $0,22 \mathrm{NS}$ \\
\hline NFO & 1,74 & 0,11 & 0,24 & $7,25^{\star *}$ & $0,47 \mathrm{NS}$ \\
\hline$H G$ & 0,27 & 0,02 & 0,01 & $27,00^{\star *}$ & $1,80 \mathrm{NS}$ \\
\hline DG & 0,64 & 0,018 & 0,011 & $58,32^{\star \star}$ & $1,63 \mathrm{NS}$ \\
\hline HDG & 0,13 & 0,004 & 0,009 & $14,44^{\star *}$ & $0,44 \mathrm{NS}$ \\
\hline LIF6 & 0,02 & 0,002 & 0,008 & $2,22 N S$ & $0,25 \mathrm{NS}$ \\
\hline TF6 & 166,40 & 7,64 & 4,13 & $40,26^{\star \star}$ & $1,85 \mathrm{NS}$ \\
\hline
\end{tabular}

$\mathrm{CM}_{\text {pop }}$ : carré moyen population; $\mathrm{CM}_{\text {int }}$ : carré moyen interaction ; NS : non significatif ; * significatif ; ** hautement significatif. $\mathrm{F}_{\text {table }}$ $(7 / 14)=2,76$ à $5 \%$ et 4,28 à $1 \%$. $F_{\text {table }}(2 / 14)=3,74$ à $5 \%$ et 6,51 à $1 \%$.

Tableau III. Résultats de l'analyse de variance à 2 critères de classification (modèle aléatoire) des différents caractères étudiés chez les populations de $M$ intertexta.

\begin{tabular}{|c|c|c|c|c|c|}
\hline & $C M_{p o p}$ & $C M_{\text {bloc }}$ & $C M_{\text {int }}$ & $\begin{array}{c}F_{p o p} 2 / 4 \\
\left(C M_{p o p} / C M_{i n t}\right)\end{array}$ & $\begin{array}{c}F_{b l o c^{2 / 4}} \\
\left(C M_{b l o d} C M_{i n t}\right)\end{array}$ \\
\hline DF1 & 55,90 & 4,93 & 1,23 & $45,44^{\star \star}$ & 3,99 NS \\
\hline DF2 & 81,74 & 12,21 & 2,94 & $27,80^{\star \star}$ & $4,15 \mathrm{NS}$ \\
\hline DF3 & 264,43 & 5,73 & 16,96 & $15,59^{\star \star}$ & 0,33 NS \\
\hline $\mathrm{DFL}$ & 311,51 & 334,44 & 78,41 & 4,26 NS & $4,26 \mathrm{NS}$ \\
\hline PRC & 43,01 & 18,01 & 3,91 & $11,00^{\star}$ & $4,60 \mathrm{NS}$ \\
\hline LO & 84,44 & 36,47 & 8,07 & $10,46^{\star}$ & $4,51 \mathrm{NS}$ \\
\hline NIO & 1,37 & 0,67 & 1,59 & 0,86 NS & 0,42 NS \\
\hline LRL & 7269,73 & 54,90 & 946,08 & $7,68^{\star}$ & $0,06 \mathrm{NS}$ \\
\hline LRX & 33430,00 & 5293,63 & 16285,83 & 2,05 NS & 0,32 NS \\
\hline NIRL & 0,67 & 1,07 & 2,81 & $0,24 \mathrm{NS}$ & $0,38 \mathrm{NS}$ \\
\hline IXF & 94,34 & 1,47 & 2,42 & $38,87^{\star \star}$ & $0,60 \mathrm{NS}$ \\
\hline NRX & 39,64 & 1,01 & 2,36 & $16,79^{\star *}$ & $0,43 \mathrm{NS}$ \\
\hline MFI & 72,05 & 2,11 & 2,44 & $29,52^{\star \star}$ & 0,86 NS \\
\hline NSG & 5,34 & 0,41 & 0,27 & $19,77^{\star \star}$ & $1,51 \mathrm{NS}$ \\
\hline NFO & 29,43 & 0,85 & 0,67 & $43,47^{\star \star}$ & $1,26 \mathrm{NS}$ \\
\hline $\mathrm{HG}$ & 1,84 & 0,003 & 0,005 & $368,00^{\star \star}$ & $0,60 \mathrm{NS}$ \\
\hline$D G$ & 0,41 & 0,02 & 0,002 & $207,50^{\star \star *}$ & $0,95 \mathrm{NS}$ \\
\hline HDG & 0,96 & 0,006 & 0,002 & $483,50^{\star \star}$ & $3,00 \mathrm{NS}$ \\
\hline LIF6 & 0,07 & 0,02 & 0,003 & $20,00^{\star *}$ & $5,21 \mathrm{NS}$ \\
\hline TF6 & 6,29 & 4,17 & 10,93 & 0,59 NS & $0,38 \mathrm{NS}$ \\
\hline
\end{tabular}

$\mathrm{CM}_{\text {pop }}$ : carré moyen population; $\mathrm{CM}_{\text {int }}$ : carré moyen interaction ; NS: non significatif ; ${ }^{*}$ significatif ; ${ }^{\star \star}$ hautement significatif. $\mathrm{F}_{\text {table }}$ $(2 / 4)=6,94$ à $5 \%$ et 18,00 à $1 \%$. 
Tableau IV. Résultats de l'analyse de variance à 2 critères de classification (modèle aléatoire) des différents caractères étudiés chez les populations des 2 espèces.

\begin{tabular}{|c|c|c|c|c|c|}
\hline & $C M_{p o p}$ & $C M_{\text {bloc }}$ & $C M_{i n t}$ & $\begin{array}{c}F_{p o p} 10 / 20 \\
\left(C M_{p o p} / C m_{\text {int }}\right)\end{array}$ & $\begin{array}{c}F_{\text {bloc }} 2 / 20 \\
\left(C M_{\text {blod }}\left(C m_{\text {int }}\right)\right.\end{array}$ \\
\hline DF1 & 59,27 & 0,81 & 2,18 & $27,18^{\star \star *}$ & 0,37 NS \\
\hline DF2 & 192,72 & 9,17 & 7,77 & $24,80^{\star *}$ & $1,18 \mathrm{NS}$ \\
\hline DF3 & 427,94 & 7,58 & 9,15 & $46,72^{\star \star}$ & 0,82 NS \\
\hline DFL & 6180,98 & 1444,93 & 220,22 & $28,06^{\star *}$ & $6,56^{*}$ \\
\hline PRC & 200,42 & 7,06 & 7,63 & $26,26^{\star *}$ & 0,92 NS \\
\hline LO & 882,83 & 147,35 & 97,65 & $9,04^{* *}$ & $1,50 \mathrm{NS}$ \\
\hline LIO & 10,40 & 0,27 & 2,08 & $5,00^{\star \star}$ & 0,13 NS \\
\hline LRL & 5042,18 & 435,99 & 353,26 & $14,27^{\star \star}$ & 1,23 NS \\
\hline LRX & 154140,27 & 12161,05 & 12229,62 & $12,60^{\star \star}$ & 0,99 NS \\
\hline NIRL & 19,74 & 3,93 & 2,75 & $7,18^{* *}$ & 1,42 NS \\
\hline IXF & 208,25 & 1,03 & 0,65 & $317,46^{\text {** }}$ & 1,83 NS \\
\hline NRX & 33,13 & 5,23 & 3,38 & $9,78^{\star \star}$ & $1,54 \mathrm{NS}$ \\
\hline $\mathrm{MFI}$ & 148,15 & 0,95 & 0,71 & $208,07^{\star \star *}$ & 1,33 NS \\
\hline NSG & 8,06 & 0,23 & 0,19 & $41,86^{* *}$ & $1,24 \mathrm{NS}$ \\
\hline NFO & 9,87 & 0,58 & 0,34 & $28,37^{\star *}$ & 1,68 NS \\
\hline$H G$ & 1,00 & 0,02 & 0,009 & $111,11^{\text {**}}$ & 2,09 NS \\
\hline$D G$ & 0,60 & 0,01 & 0,01 & $55,05^{* *}$ & 0,90 NS \\
\hline HDG & 0,38 & 0,001 & 0,007 & $55,28^{\star *}$ & $0,14 \mathrm{NS}$ \\
\hline LIF6 & 0,05 & 0,002 & 0,008 & $7,25^{\star \star}$ & 0,34 NS \\
\hline TF6 & 117,82 & 8,41 & 5,42 & $21,73^{\star \star}$ & $1,55 \mathrm{NS}$ \\
\hline
\end{tabular}

$\mathrm{CM}_{\text {pop }}$ : carré moyen population; $\mathrm{CM}_{\text {in }}$ : carré moyen interaction ; NS : non significatif ; * significatif ; ** hautement significatif. $\mathrm{F}_{\text {table }}$ $(10 / 20)=2,35$ à $5 \%$ et 3,37 à $1 \%, F_{\text {table }}(2 / 20)=3,49$ à $5 \%$ et 5,85 à $1 \%$.

(DFL, NIO, LRX, NIRL et TF6) sur 20 ne présentent pas de différences significatives (tableau II). Enfin, l'analyse de variance appliquée à l'ensemble des 11 populations regroupant les 2 espèces met en évidence un effet population hautement significatif pour tous les caractères utilisés (tableau IV).

Les variations importantes observées entre les différents paramètres témoignent d'une hétérogénéité phénotypique importante entre les populations. Cette variabilité est de nature génétique puisque les populations ont été cultivées dans un milieu relativement homogène.

\section{Analyse de la diversité globale des populations des 2 espèces}

\section{Les populations de $M$ ciliaris}

\section{Matrice des corrélations}

Le tableau $\mathrm{V}$ représente la matrice des corrélations entre les 20 caractères étudiés. Son examen montre des corrélations positives entre les caractères DF1 et DF2 et DF3, DFL et PRC, LO et NIO, LRL et LRX, IXF et MFI, HG et DG, HG et HDG. Par conséquent, il nous a paru utile de réduire le nombre de caractères, en écartant ceux qui présentent une forte corrélation, ce qui permet de diminuer les redondances qui peuvent exister entre elles lors des analyses multivariées.

\section{Absorption de la variabilité par les 2 premiers axes et leur signification par rapport aux variables}

Le tableau VI, découlant de l'analyse canonique discriminante, donne les pourcentages d'inertie et les paramètres qui contribuent le plus à la définition des 2 premières composantes canoniques. Ces dernières rendent compte de la plus grande partie de la dispersion, puisqu'elles absorbent $79,15 \%$ de la variabilité totale. La représentation graphique de l'analyse canonique discriminante dans le plan 1-2 (fig 2) révèle un chevauchement plus ou moins marqué entre les polygones de dispersion représentant les 8 populations. La composante canonique 1 oppose les populations (Biz) et (Meb) qui possèdent un étalement rapide des premières feuilles et une mise à fleur tardive (131 j en moyenne), aux populations (Seb), (Jen), (Tun), (Bej) et (Kor). Par ailleurs, la popu- 


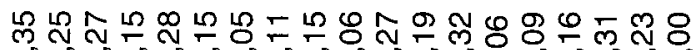
ípió0

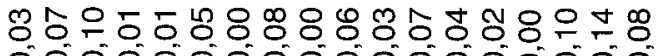
100

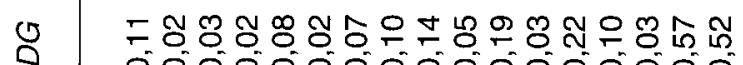

全

0

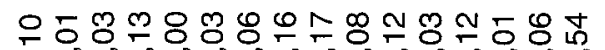

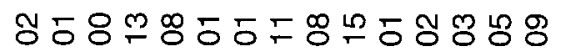
o.

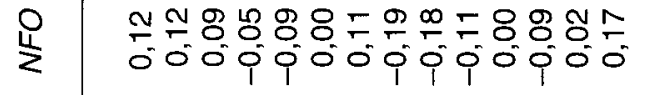

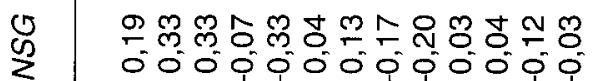

$\gtrless$ o0

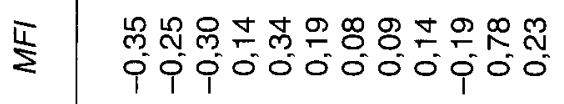

กำ ำㄴำำ

$\stackrel{4}{\star}$

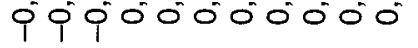

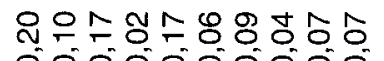

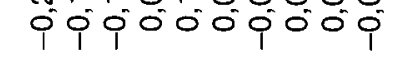

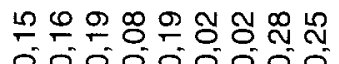

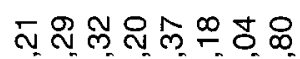

ipiónó

$\stackrel{1}{a}$

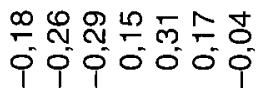

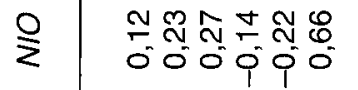

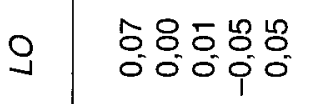

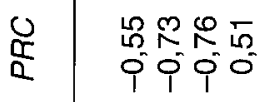

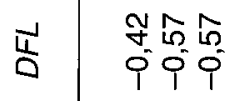

บับ

殅 ?

$\frac{0}{2} \frac{1}{0}$ 
Tableau VI. Pourcentages d'inertie (\%lnt) et définition des axes de l'analyse canonique discriminante pour les 8 populations de $M$ ciliaris.

\begin{tabular}{lrr}
\hline Axes & \multicolumn{1}{l}{2} \\
& & \multicolumn{1}{l}{} \\
\%Int & 58,94 & 20,20 \\
& & \\
Variables & \multicolumn{1}{l}{ Coe } & \multicolumn{1}{l}{ Coe } \\
& & \\
DF3 & & \\
DFL & 0,970 & 0,097 \\
LO & $-0,561$ & $-0,151$ \\
LRL & $-0,048$ & 0,182 \\
NIRL & $-0,330$ & $-0,172$ \\
NRX & 0,217 & 0,108 \\
MFI & 0,293 & 0,122 \\
NSG & $-0,437$ & 0,324 \\
NFO & 0,337 & 0,230 \\
DG & 0,097 & $-0,093$ \\
HDG & $-0,031$ & $-0,882$ \\
L1F6 & $-0,059$ & 0,439 \\
TF6 & 0,094 & 0,170 \\
& $-0,419$ & 0,699 \\
\hline & & \\
\hline
\end{tabular}

Coe : coefficient canonique des variables. lation $(\mathrm{Mjb})$ semble occuper une situation intermédiaire. II est important de signaler que (Meb) et $(\mathrm{Biz})$ sont situées le plus au nord, tandis que les populations (Seb), (Jen), (Tun), (Bej), (Mjb) et (Kor) sont localisées le plus au sud dans l'aire prospectée. Toutefois, le passage d'un groupe à un autre semble se faire sans discontinuité brutale, ce qui témoigne d'une gamme de variabilité relativement continue.

La composante canonique 2 représente $20,20 \%$ seulement de la variation totale. Elle permet de distinguer les populations (Meb), (Seb) et (Tun) des autres (Jen, Biz, Mjb, Kor et Bej) marquées par un diamètre de la gousse relativement important (DG élevé) et par une grande taille de la foliole terminale de la sixième feuille (TF6).

Les distances de Mahalanobis $\left(D^{2}\right)$ calculées entre les différentes populations sont toutes significatives. Le dendrogramme établi à partir de ces distances (fig 3) fait ressortir 2 groupes de populations distants de 36,58 : le premier est formé par les populations de (Biz) et (Meb), le second est constitué par le reste des populations. Dans ce dernier groupe, les plus faibles distances de Mahalanobis sont de 5,02 et 7,31. Elles séparent respectivement les couples de populations (Seb, Tun) et (Kor, Jen).

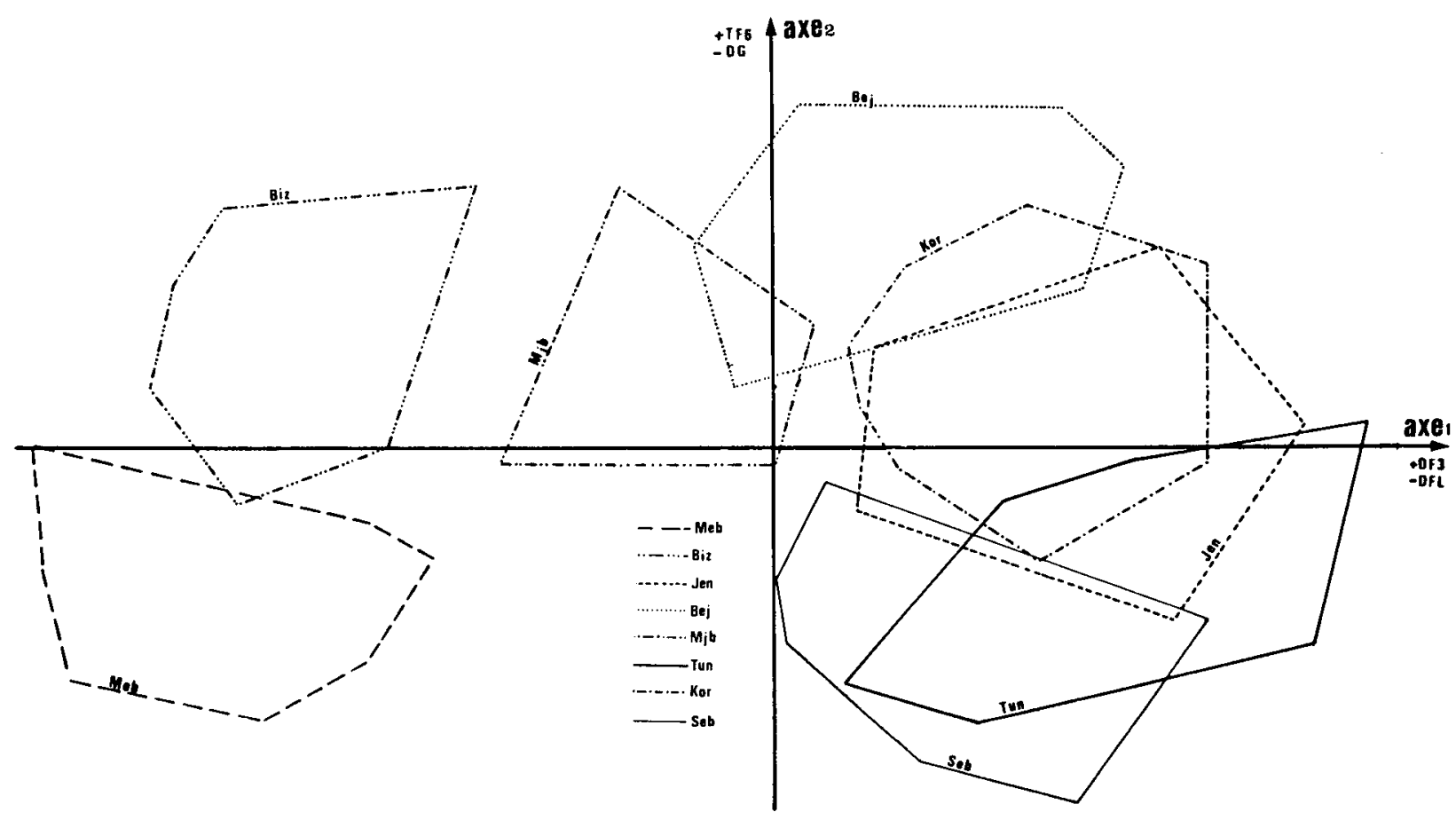

Fig 2. Représentation graphique des polygones de dispersion des 8 populations de $M$ ciliaris dans une analyse canonique discriminante. 


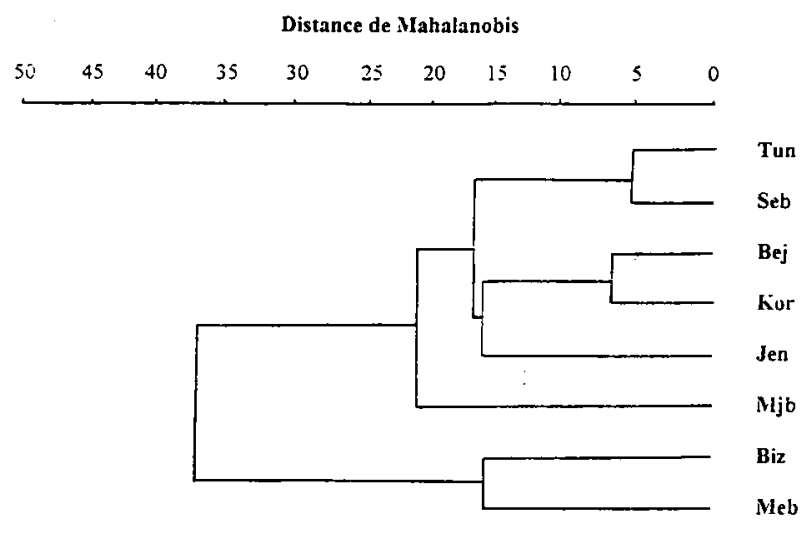

Fig 3. Dendrogramme de classification des populations de $M$ ciliaris établi à partir des distances de Mahalanobis.

\section{Les populations de $M$ intertexta}

\section{Matrice des corrélations}

La majorité des caractères corrélés chez $M$ ciliaris (sauf pour les paramètres DFL et PRC) se trouvent aussi chez $M$ intertexta. Toutefois, on note ici une forte corrélation entre HG et HDG $(0,85)$ (tableau VII).

\section{Absorption de la variabilité par les 2 premiers axes et leur signification par rapport aux variables}

Le tableau VIII traduit la signification des 2 permières composantes canoniques, qui absorbent $100 \%$ de la variabilité totale. Les polygones de dispersion des 3 populations (fig 4), représentés sur le plan défini par les axes 1-2, sont nettement séparés les uns des autres, ce qui témoigne d'une hétérogénéité relativement importante entre les 3 populations analysées. Selon l'axe 1 qui contribue à $81,38 \%$ de l'intertie totale, la population de Sedjnane (Sed) se distingue des 2 autres (Aïd et Mat) par un nombre faible de fleurs par inflorescence (2-7 fleurs), par des gousses relativement allongées (HDG élevé), par un nombre élevé de feuilles sur l'axe orthotrope et par une initiation foliaire plus précoce. Quant à la population de (Aïd), elle semble être bien définie sur l'axe 2 qui absorbe $18,62 \%$ de la variabilité. Elle est caractérisée par des gousses à diamètre (DG) et à nombre de spires (NSG) importants.

Les distances de Mahalanobis $\left(\mathrm{D}^{2}\right)$, calculées entre les 3 populations prises 2 à 2 , se sont avé- rées toutes significatives. Leur représentation sous forme d'un dendrogramme (fig 5) montre que les populations (Aïd) et (Mat) sont les plus proches $\left(D^{2}=15,46\right)$, et s'éloignent ensemble de 44,50 de la population (Sed).

\section{Les populations des 2 espèces}

$M$ ciliaris et $M$ intertexta présentent, particulièrement à l'état végétatif, des ressemblances morphologiques importantes (Lesins et al, 1971 ; Lesins et Lesins, 1979 ; Small et Jomphe, 1989). Pour élucider le degré de similitude entre ces 2 taxons (du moins par les caractères retenus) et afin de mieux préciser leurs définitions spécifiques, les 11 populations représentant les 2 espèces ont été regroupées dans une même analyse canonique discriminante.

\section{Matrice des corrélations}

La matrice des corrélations entre les 20 caractères étudiés est portée sur le tableau IX. L'analyse de ce dernier révèle globalement les mêmes corrélations décrites pour les 2 espèces prises séparément. Ainsi, les caractères DF1 et DF2 et DF3, DFL et PRC, LO et NIO, LRL et LRX, MF1 et IXF sont corrélés positivement.

\section{Absorption de la variabilité par les 2 premiers axes et leur signification par rapport aux variables}

Le tableau $X$ regroupe les pourcentages d'inertie et la définition des 2 premiers axes de la canonique discriminante qui absorbent dans leur ensemble $79,92 \%$ de la variabilité totale.

Le premier axe, défini par le nombre moyen de fleurs par inflorescence (MFI) et par les dimensions de la gousse (HG et HDG), oppose les populations de $M$ intertexta (situées du côté positif) aux populations de $M$ ciliaris (situées du côté négatif) (fig 6). Ces dernières se distinguent donc par un nombre faible de fleurs par inflorescence et par des gousses de grande taille. La nette séparation des populations des 2 espèces selon l'axe 1, corrélé aux caractères MFI, HG et HDG, souligne l'importance de ces marqueurs morphologiques, non souvent considérés par les botanistes dans l'identification spécifique des 2 taxons (Pottier-Alapetite, 1979 ; Lesins et Lesins, 1979 et Zouari, 1981).

Par ailleurs, la population de $M$ intertexta (Sed), s'isolant de son groupe d'origine et de celui de $M$ ciliaris, se singularise par sa position 


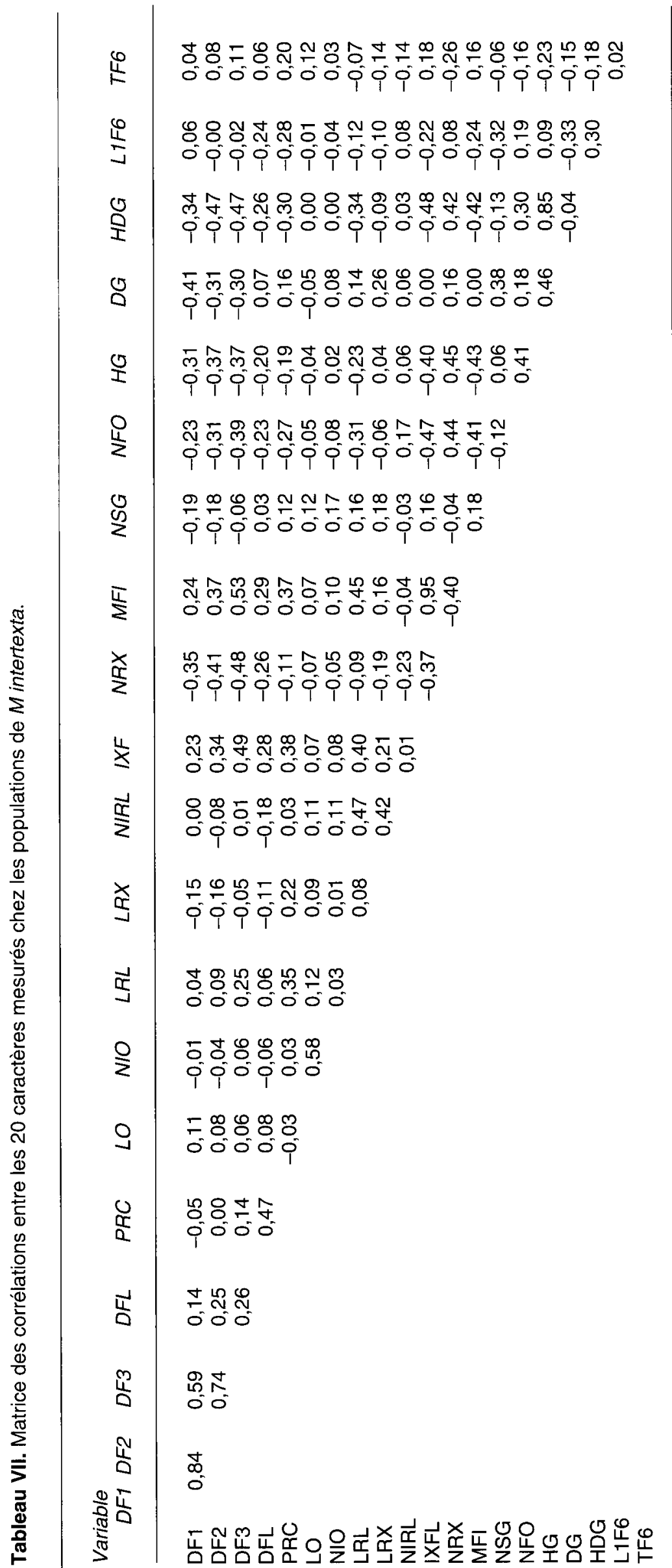




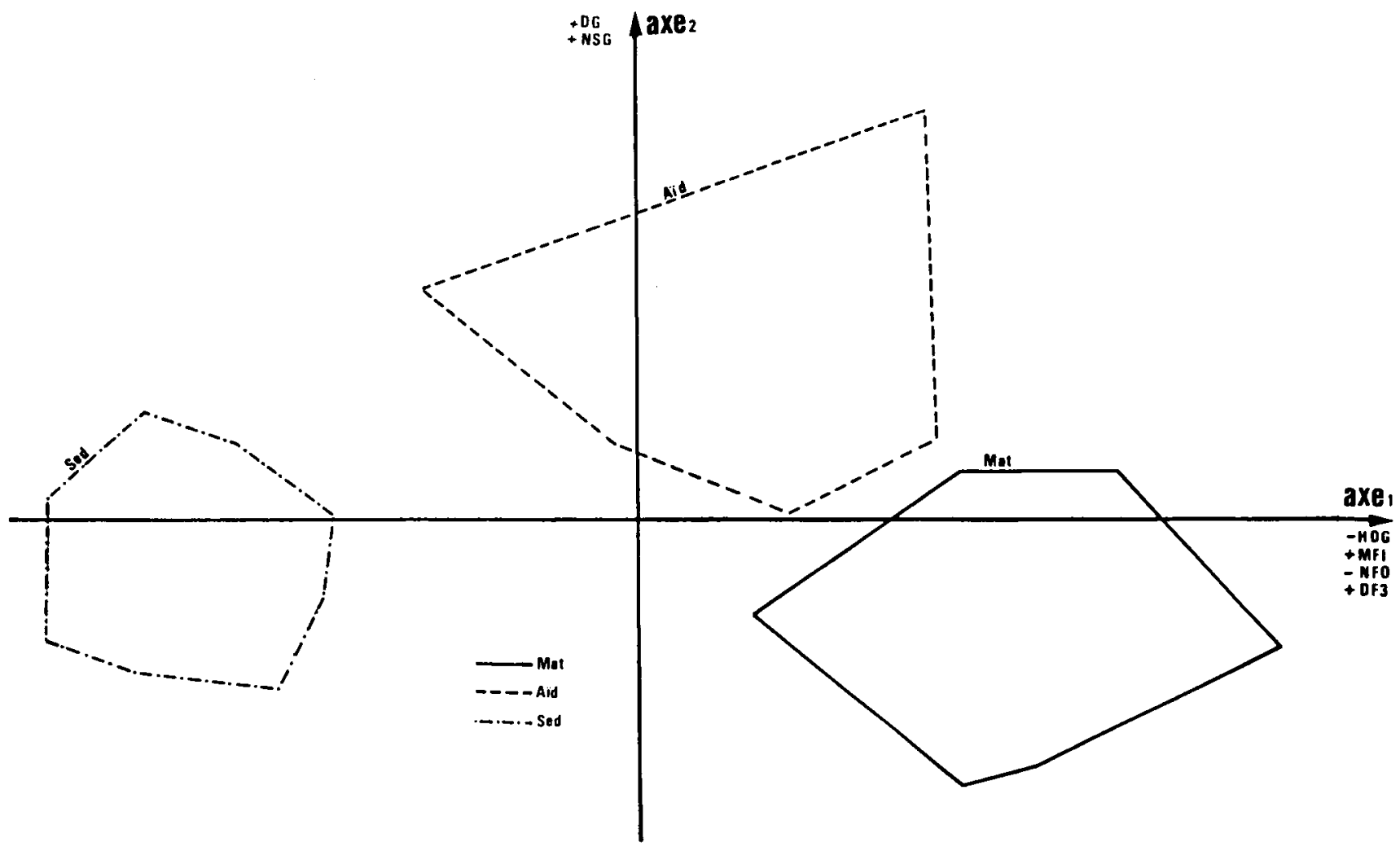

Fig 4. Représentation graphique des polygones de dispersion des 3 populations de $M$ intertexta dans une analyse canonique discriminante.

Tableau VIII. Pourcentages d'inertie (\%Int) et définition des axes de la canonique discriminante des 3 populations de $M$ intertexta.

\begin{tabular}{|c|c|c|}
\hline Axes & 1 & 2 \\
\hline \%Int & 81,38 & 18,62 \\
\hline Variables & Coe & Coe \\
\hline DF3 & 0,682 & $-0,258$ \\
\hline DFL & 0,347 & 0,260 \\
\hline PRC & 0,410 & 0,331 \\
\hline LO & 0,115 & 0,159 \\
\hline LRL & 0,439 & 0,202 \\
\hline NIRL & $-0,053$ & $-0,066$ \\
\hline NRX & $-0,659$ & 0,085 \\
\hline $\mathrm{MFI}$ & 0,807 & 0,172 \\
\hline NSG & 0,048 & 0,681 \\
\hline NFO & $-0,780$ & $-0,034$ \\
\hline DG & $-0,203$ & 0,846 \\
\hline HDG & $-0,901$ & $-0,172$ \\
\hline L1F6 & $-0,288$ & $-0,585$ \\
\hline TF6 & 0,241 & $-0,004$ \\
\hline
\end{tabular}

Coe: coefficient canonique des variables. intermédiaire entre les 2 ensembles de populations représentant les 2 espèces.

Le deuxième axe, représentant $17,82 \%$ de la variation totale, reflète une dispersion des populations de $M$ ciliaris analogue à celle décrite sur la figure 2.

Les distances de Mahalanobis sont toutes significatives, même entre les populations d'un même taxon. Le dendrogramme établi à partir de ces distances (fig 7) révèle des regroupements de populations qui rappellent ceux constatés par la représentation graphique de la canonique discriminante (fig 6). Les populations de $M$ ciliaris sont toujours groupées ensemble

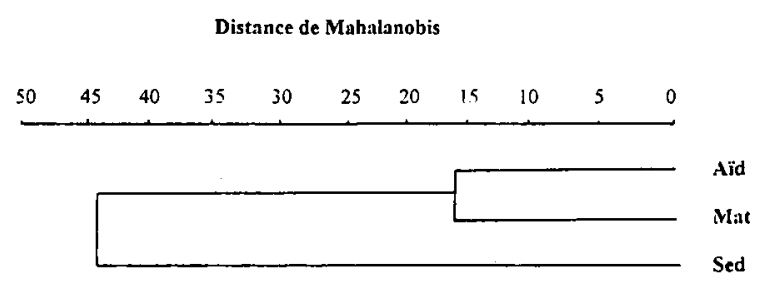

Fig 5. Dendrogramme de classification des populations de $M$ intertexta établi à partie des distances de Mahalanobis. 


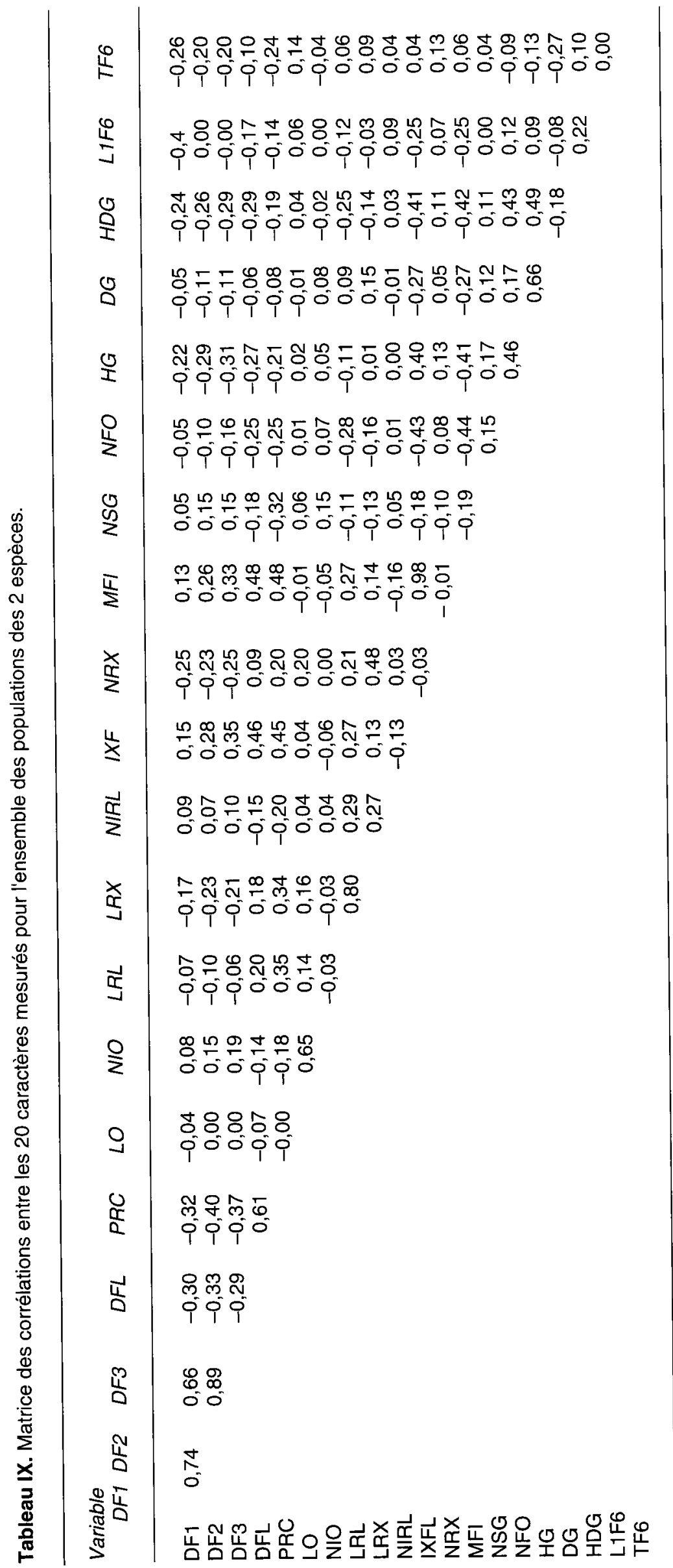




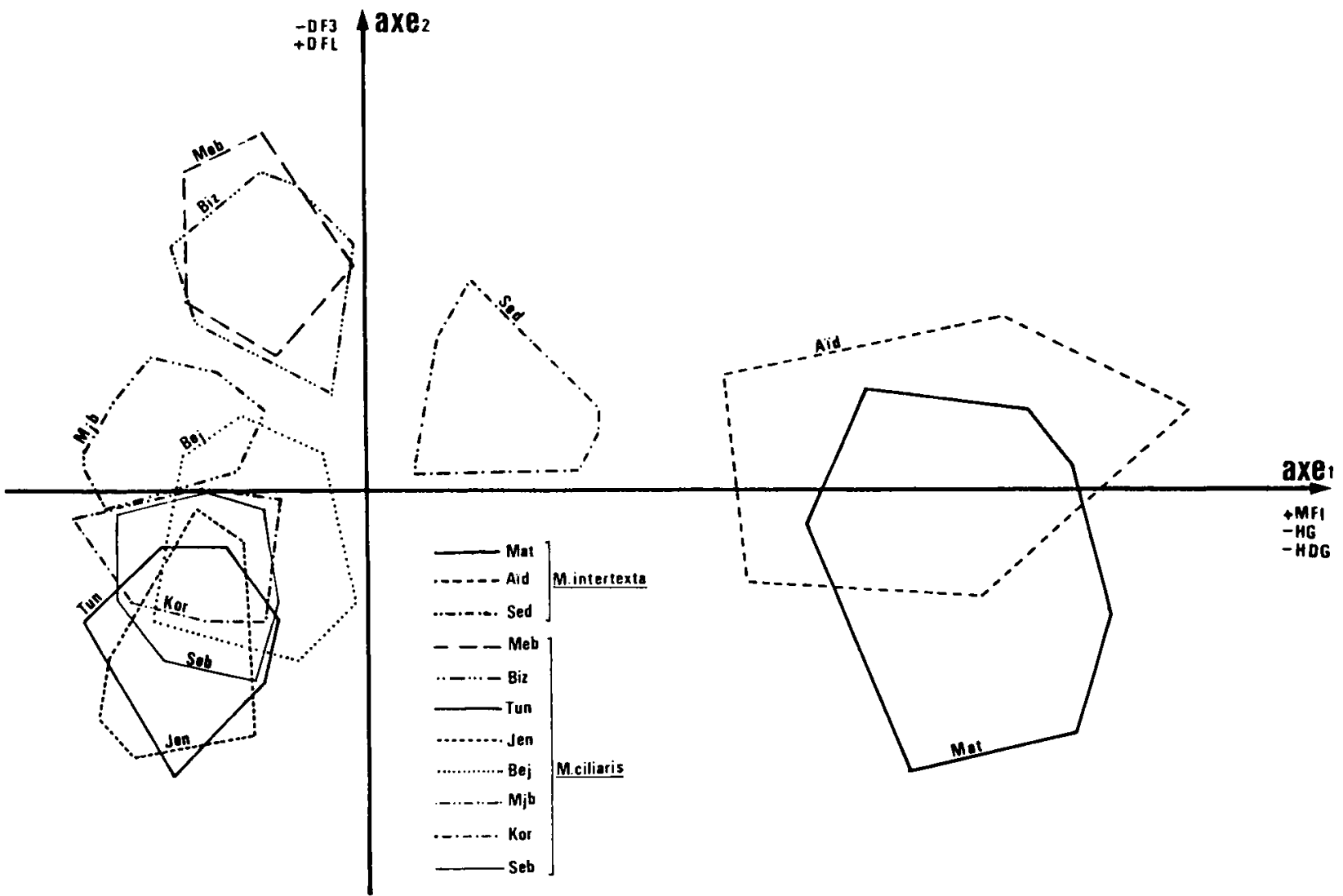

Fig 6. Représentation graphique des polygones de dispersion des populations des 2 espèces ( $M$ ciliaris et $M$ intertexa) dans une analyse canonique discriminante.

Tableau X. Pourcentages d'inertie (\%Int) et définition des axes de l'analyse canonique discriminante pour l'ensemble des populations des 2 espèces.

\begin{tabular}{|c|c|c|}
\hline Axes & 1 & 2 \\
\hline$\%$ Int & 62,10 & 17,82 \\
\hline Variables & Coe & $\mathrm{Coe}$ \\
\hline DF3 & 0,363 & $-0,886$ \\
\hline DFL & 0,422 & 0,641 \\
\hline LO & $-0,024$ & 0,036 \\
\hline LRL & 0,289 & 0,193 \\
\hline NIRL & $-0,154$ & $-0,189$ \\
\hline NRX & $-0,058$ & 0,354 \\
\hline MFI & 0,986 & 0,031 \\
\hline NSG & $-0,237$ & $-0,257$ \\
\hline NFO & $-0,505$ & $-0,061$ \\
\hline$H G$ & $-0,685$ & 0,155 \\
\hline HDG & $-0,601$ & 0,196 \\
\hline L1F6 & $-0,283$ & $-0,081$ \\
\hline TF6 & 0,058 & 0,404 \\
\hline
\end{tabular}

Coe: coefficient canonique des variables. avec des distances variant de 4,65 (pour le couple de populations Seb et Tun) à 32,84 entre les 2 groupes (Biz, Meb) et (Seb, Tun, Jen, Kor, Bej, Mjb). Dans le cas de $M$ intertexta, les populations (Aïd) et (Mat) sont également associées $\left(\mathrm{D}^{2}=7,80\right)$, mais la population Sed se regroupe plutôt avec les populations de $M$ ciliaris avec une distance de 35,57 .

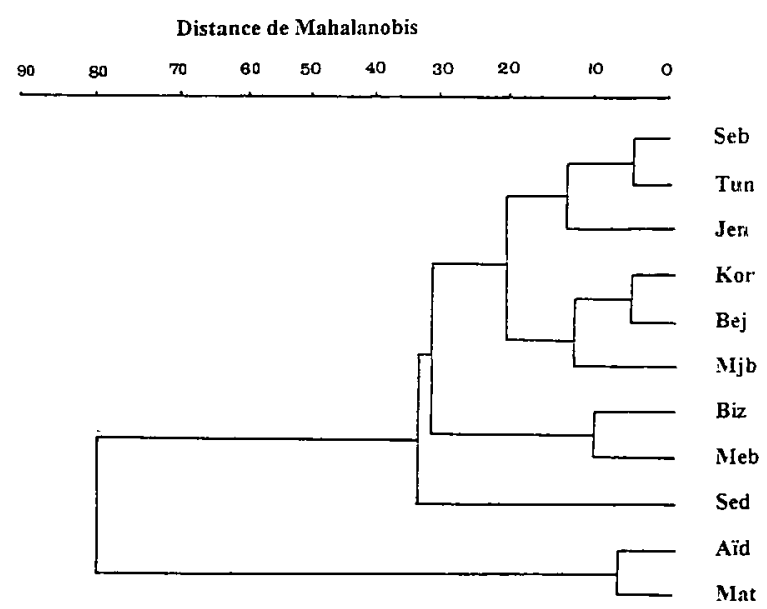

Fig 7. Dendrogramme de classification des populations des 2 espèces, établi à partir des distances de Mahalanobis. 


\section{DISCUSSION ET CONCLUSIONS}

L'analyse canonique discriminante, appliquée à 20 caractères morphologiques, a permis de définir les grands axes de la variabilité génétique des populations de $M$ ciliaris et $M$ intertexta et de prévoir les divergences morphologiques entre les 2 espèces.

Les dimensions de la gousse (DG, HG, HDG), le nombre de spires par gousse (NSG), le nombre de feuilles sur l'axe orthotrope (NFO) et la précocité de la floraison (DFL, PRC), et de l'initiation foliaire (DF1, DF2, DF3) permettent de discriminer plus ou moins bien les populations au sein de la même espèce.

La variabilité génétique importante au sein de chaque espèce pourrait être également favorisée par le mode de reproduction préférentiellement autogame rencontré chez les 2 taxons. L'autogamie favoriserait l'hétérogénéité entre les populations, elle permettrait ainsi une meilleure adaptation au milieu (Hamrick et Godt, 1990 ; Clegg et al, 1992). En outre, la diversité des structures génotypiques rencontrées dans chacune des 2 espèces contribuerait à la colonisation de milieux divers et offrirait ainsi de nombreuses possibilités de sélection et d'amélioration au sein de chaque taxon.

L'analyse des populations des 2 espèces prises simultanément souligne l'existence de 2 compartiments spécifiques $M$ intertexta et $M$ ciliaris. La distinction entre les 2 taxons repose particulièrement sur le nombre de fleurs par inflorescence (MFI) et les dimensions de la gousse (HG et HDG). L'utilisation de ces caractères dans la description botanique des 2 espèces pourrait mieux préciser les révisions spécifiques de Lesins et al (1971) et Small et Jomphe (1989). Toutefois, dans notre étude, une population de $M$ intertexta originaire de Sedjnane (Sed) présente un ensemble de caractères (MFI, HG et HDG) qui contribuent à son rapprochement des populations de $M$ ciliaris. La position intermédiaire de cette population suggère l'éventualité d'hybridation entre les 2 espèces, comme cela a été signalé par Lesins et al (1971) et Small et Jomphe (1989). Des caractères relatifs à la biologie florale semblent confirmer cette hypothèse. En effet, cette population présente un taux de viabilité des grains de pollen relativement faible $(75 \%)$ par rapport à celui noté chez les autres $(98 \%)$. De plus, cette population manifeste une baisse de production de gousses formées par fleur ( $11 \%$ en moyenne contre $23 \%$ chez les autres populations). Cependant, les résultats présentés ici ne nous permettent pas de conclure quant au statut hybride ou non de cette population.

Une analyse cytogénétique et une étude de polymorphisme enzymatique ainsi que de celui de I'ADN pourraient apporter des éléments d'information quant à la nature de cette population.

\section{RÉFÉRENCES}

Abdelkefi A, Boussaïd M, Marrakchi M (1992) Prospection et inventaire des espèces spontanées du genre Medicago $L$ en Tunisie. Actes du colloque international "Complexe d'espèces, flux de gènes et ressources génétiques des plantes». Paris, 8-10 janvier 1992

Baâtout H, Marrakchi M, Combes D (1991) Genetic divergence and allozyme variation within and among populations of Hedysarum spinosissimum subsp capitatum and subis spinosissimum (Papilionaceae). Taxon 40, 239-252

Clegg MT, Epperson BK, Brown AHD (1992) Genetic diversity and reproductive system. In: Reproductive biology and plant breeding ( $\mathrm{Y}$ Dattée, $\mathrm{C}$ Dumas, A Gallais, eds), Springer-Verlag, Berlin, 331-323

Damerval C (1983) Comparaison de 6 espèces de luzernes annuelles à l'aide de caractères biométriques et enzymatiques. agronomie 3, 10, 971-982

Elmossadik A (1991) Analyse de la variabilité génétique des écotypes marocains du genre Medicago. Implication en gestion des ressources phytogénétiques. Thèse $3^{e}$ cycle, université Mohamed $V$, fac sciences de Rabat, $132 \mathrm{p}$

Fisher RA (1936) The use of multiple measurements in taxonomic problems. Ann Eugen, 7, 179-188

Gintzburger G, Prosperi JM (1987) D'autres luzernes annuelles. Bulletin FNAMS «Semences», $101 \mathrm{p}$

Hamrick JL, Godt NJ (1990) Allozyme diversity in plant species. In: Plant population genetics, breeding and genetic resources (AHD Brown, MT Clegg, AL Kahler, BS Weir, eds). Sinauer Associates, Sunderland, MA, 43-63

Hebrant F (1974) Problèmes de discrimination dans le cas de plusieurs populations. Biom Praxim IXV, 3-4

IBPGR (1991) Descripteurs pour Medicago annuelles. International board for plant genetic ressources, Rome, $33 \mathrm{p}$

Lesins K, Singh SM, Erac A (1971) Relationship of taxa in the genus Medicago as revealed by hybridization. Section Intertextae. Can J Genet Cytol 13, 335-343

Lesins K, Lesins I (1979) Genus Medicago (Leguminosae), a taxogenetic study. W Junk bv, the Hague

Pottier-Alapetite G (1979) Flore de la Tunisie. Angiospermes dicotylédones. Apétales-dialypétales. Imp Off République tunisienne, $542 \mathrm{p}$ 
Rao CR (1972) Recent trends of research works in multivariate analysis. Biometrics 28, 3-22

Robert R, Sarr A (1991) Multivariate analysis recombination between wild and cultivated genomes within the primary gene pool of pearl millet (Pennisetum thyphoides). Genome 35, 208-219

Rouz S (1991) Analyse morphologique et productive de divers écotypes de Medics. Mémoire $2^{e}$ cycle INAT, Tunisie, $74 \mathrm{p}$

SAS (1990) SAS user's guide: SAS STAT, SAS BASIC. Version 6, fourth edition. SAS circl, Box 8000 . Cary, NC 27512-8000, Cary, NC: SAS Institute Inc

Semple JC, Chmielewski JG, Leeder C (1991) A multivariate morphometric study and revision of Aster subg Doellingeria sect Triplopappus (Compositae:
Astereae): the Aster umbellatus complex. Can J Bot 69, 256-276

Singh SP, Gutierrez JA, Molina A, Urrea C, Gepts P (1991) Genetic diversity in cultivated common Bean. II. Marker-based analysis of morphological and agronomic traits. Crop Sci 31, 23-29

Small E, Jomphe $M$ (1989) A synopsis of the genus Medicago (Leguminosae). Can J Bot 67, 3260-3294

Small E, Fawzy M (1992) Morphologic variation in the Medicago monantha complex. Can J Bot 70, 12921301

Zouari $T$ (1981) Flore et écologie taxinomique de la Tunisie. Dicotyledones VII : Dialypétales Calyciflores. Thèse doctorat fac Saint-Jérome, AixMarseille III, $215 \mathrm{p}$ 